\title{
Asynchronous updates can promote the evolution of cooperation on multiplex networks
}

\author{
James M. Allen ${ }^{\mathrm{a}, \mathrm{b}, *}$, Rebecca. B. Hoyle ${ }^{\mathrm{c}}$ \\ ${ }^{a}$ Department of Mathematics, University of Surrey, Guildford, GU2 7XH, UK \\ ${ }^{b}$ Evolution and Resilience of Industrial Ecosystems, University of Surrey, Guildford, GU2 7XH, UK \\ ${ }^{c}$ Mathematical Sciences, University of Southampton, SO17 1BJ, UK
}

\begin{abstract}
We study the importance to the frequency of cooperation of the choice of updating strategies in a game played asynchronously or synchronously across layers in a multiplex network. Updating asynchronously in the public goods game leads to higher frequencies of cooperation compared to synchronous updates. How large this effect is depends on the sensitivity of the game dynamics to changes in the number of cooperators surrounding a player, with the largest effect observed when players payoffs are small. The discovery of this effect enhances understanding of cooperation on multiplex networks, and demonstrates a new way to maintain cooperation in these systems.

Keywords:

Cooperation, multiplex networks, public goods game

PACS: 89.75.Fb, 87.23.Ge, 02.50.Le
\end{abstract}

\section{Introduction}

In many social systems a social dilemma exists where what is good for the individual is not necessarily good for the group, and so explaining why in both laboratory [1, 2] and field [3, 4] experiments participants are found to cooperate (act in the interests of the group) more frequently than would be expected for purely rational self-interested players has proved a challenge to evolutionary biology and sociology. The prevalence of cooperation between unrelated members of a population in both biological and human social systems has been the subject of a long history of study, with a number of key insights over the past few decades [5].

One of the central tools used to understand the resolution of social dilemmas and the evolution of cooperation is game theory [6]. Here each player can choose from a number of strategies with a payoff, dependent on their own strategy and those chosen by their opponents, indicating to each player how well they are performing. Playing the prisoner's dilemma (PD) on a lattice network (where the network defines those that each player plays against) cooperation has been shown to increase compared to the unstructured

*j.m.allen@surrey.ac.uk 
case [7] by cooperators forming clusters that are resistant to exploitation by surrounding defectors, an effect known as network reciprocity. Developing these ideas the evolution of cooperation on networks received intense subsequent study, with the effect on cooperation of network structure $[8,9,10,11]$, the type of game [12], the rules used to update the strategy [13], additional strategies such as punishment and loners $[14,15,16]$ and diversity between players $[17]$ investigated. In each case it was found that added heterogeneity introduces higher levels of cooperation, as cooperators are able to seed clusters more easily and boost the effect of network reciprocity.

In scenarios such as electricity power networks and networks of air travel it is more accurate to describe a system as a combination of networks than a single one $[18,19,20,21,22]$. These multilayered systems can take two forms: either interdependent networks, where two separate networks are connected through a number of between-network edges, or multiplex networks, where the nodes on each layer represent the same entity, but each layer represents a different aspect of the system [23].

How the amount of cooperation on a multilayered system varies has been studied in a number of cases, from interdependent networks of different topologies [24, 25] to lattices with weighted payoffs between the layers [26, 27] and weighted fitnesses [28], probabilistic connections between layers [29, 30], different imitation rules between layers [31] and memory [32]. In each of these cases cooperation is enhanced by the addition of extra networks caused by strategies on each layer supporting each other against exploitation by defectors, and there is a peak interdependence between the two layers due to maximum heterogeneity in the system, even when the games played on each network are different [33].

The dynamics of games have also been studied on multiplex networks [34]. These are networks with more than one layer, where the nodes on each layer represent the same player, but the connections between them are not necessarily the same. On these networks players can play different strategies on each layer, but their payoffs are summed across all layers. Cooperation was found to be increased compared to the single layer when games are played on random network multiplexes due to the ability of players to play a differing strategy on each layer $[35,36]$. These "incoherent" players are able to maintain cooperation when it would usually have disappeared in the single layer system. The effect of separating the networks on which players calculate their payoffs and those on which they update their strategies onto different layers has been explored [37], as has how the assortativity of the degrees between the networks affects the final frequency of cooperation, what happens if the game played on each layer of the multiplex is different [38], and if players imitate those that they are most similar to [39]. Zhang et al. [40] found the analytical conditions for cooperation on a multiplex by studying structured groups across a two-layer network, where cooperation was found to be maximised when intermediate levels of migration between groups was implemented.

Despite the existing work discussing cooperation in multilayered networks, more needs to be done in order to understand exactly what is important in these systems for cooperation to be maintained, and how cooperation can be encouraged in order to offer solutions to social dilemmas. One assumption that is made 
in a number of previous models is about how frequently the nodes on each layer update themselves. It has been found $[13,41]$ that the proportion of nodes that update their strategies on a network before the payoff of each player is recalculated can alter the amount of cooperation in the system. What has not been considered in the case of multilayered networks is whether updating each of the nodes across all layers compared to just a single layer at a time affects the final amount of cooperation in the system. Whether a player updates their strategy on all layers of the system at the same time or separately will depend strongly on the system that is to be modelled. It is therefore important to know whether this has any effect on the final frequency of cooperation in the system. This is the question that we will address in this article.

\section{Mathematical Model}

In order to study the spread of cooperation on the multiplex network the public goods game (PGG) is played by each of the nodes. In this game there are two possible strategies, either cooperation or defection. In the PGG the players are divided into groups before donating a certain amount to the groups that they are members of. The total that has been donated is then multiplied up by an enhancement factor, before being divided between all of the members of that group. When playing the PGG on a network the groups are defined to be those players that are connected to a common node. So, on each layer the player plays the game in $k+1$ groups, where $k$ is the degree of the player on that particular layer. In the PGG, player $i$ donates an amount $c_{i}$, and so the payoffs are given by

$$
\begin{gathered}
P_{D}^{i}=r \frac{\sum_{j=1}^{N_{C}} c_{j}}{G} \\
P_{C}^{i}=r \frac{\sum_{j=1}^{N_{C}} c_{j}+c_{i}}{G}-c_{i}
\end{gathered}
$$

where $P_{C, D}^{i}$ is the payoff of player $i$ playing as a cooperator or a defector respectively, $G$ is the number of players in the group, $N_{C}$ is the number of cooperators in the group, $r$ is the enhancement factor (the return on the investment to the group) and the last term in equation (2) represents the donation by the cooperators to the public good. The final payoff for each player is found by summing across all of the groups in which they play across all of the layers. We will study two possible versions of the PGG: fixed cost per group (FCG) when each player donates one unit to whichever group they are playing the game in e.g. $c_{i}=1$ for all groups, or fixed cost per individual (FCI) where each player has only a single unit to donate and divides this between all of the groups in which it plays e.g. $c_{i}=\frac{1}{k_{i}+1}$ where $k_{i}$ is the degree of node $i$.

Initially each player on each layer is assigned a strategy (cooperate or defect) at random, which need not be the same on every layer. Each node plays the PGG against their neighbours on each layer, with each player's payoff accumulated across all layers. Once the payoffs have been calculated the players have an opportunity to update their strategy through imitation. In general, it makes sense for a player to imitate 
those that are performing better. Therefore on selected layers each player chooses a neighbour at random and compares payoffs, imitating the neighbour's strategy with probability calculated below.

We study the game on a multiplex consisting of two Erdős-Rényi random networks of size $N$ nodes. These networks are formed independently on each layer by creating an edge between two nodes with probability $\frac{\langle k\rangle}{N-1}$, where $\langle k\rangle$ is the mean degree of each node. The final payoff of player $i$ is calculated by summing the payoffs for each layer e.g. $P_{i}=\sum_{j=1}^{L} p_{i j}$ where $L$ is the total number of layers and $p_{i j}$ is the payoff of player $i$ on layer $j$. A player is more likely to imitate another's strategy if that player has a higher payoff, and the probability of imitation should be proportional to the payoff difference. Therefore, in common with many other studies of cooperation on networks $[8,9,10,11]$ player $i$ will imitate the strategy of neighbour $j$ with probability

$$
P\left(s_{i} \rightarrow s_{j}\right)=\frac{1}{1+e^{-\frac{P_{j}-P_{i}}{\beta}}}
$$

where $\beta$ is a parameter that defines how responsive the player is to their neighbour's payoff. Here $\beta=0$ leads to deterministic dynamics where a player will always imitate the strategy of a player with a larger payoff, and $\beta \rightarrow \infty$ leads to players imitating their neighbours strategy at random, with no consideration of their respective payoffs.

In this article we investigate the effect of updating the players strategies either asynchronously or synchronously across layers on the final frequency of cooperation. In the asynchronous case, after the payoffs of every player have been calculated each player selects one layer at random on which to update their strategy, and then a random neighbour on that layer to compare total payoffs. When updating synchronously, each player selects a neighbour to imitate on each layer (not necessarily the same neighbour on each). On this multiplex, each player is allowed to play a different strategy on each layer even though they are the same individual. Examples of a multiplex network, where a single player and its strategy on each layer are highlighted, and examples of each rule are shown in figure 1, where the nodes in the hollow squares are those updating their strategy. In order to compare the different rules the asynchronous update rule will update twice per time step and the synchronous rule just once so that each node updates the same number of times on average.

We study how updating the player strategies either asynchronously or synchronously affects the final frequency of cooperation for both the PGG and the PD. In the results presented below for each iteration and for each rule a new network and initial conditions are generated. In order to ensure that the system has reached equilibrium each node is updated 50,000 times on average for each run. The final frequency of cooperation is then averaged over the last 5000 complete updates of the system. Results for each simulation are plotted as points and the mean of all of the simulations is shown by a line. All results presented in this article (unless otherwise stated) are for $m=2$ layer multiplex networks with $N=1000$ nodes on each layer. 


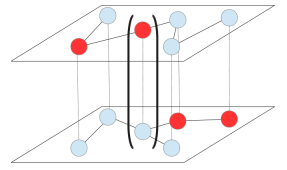

(a) An example of a multiplex network

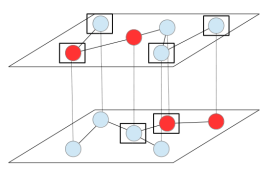

(b) Asynchronous update

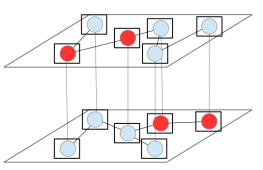

(c) Synchronous update

Figure 1: An example of a multiplex network (1a) with a single player and their strategy on each layer indicated, the asynchronous update (1b) and the synchronous update (1c) rules. The nodes that are contained in squares are those selected to update their strategy in that round. Red dots denote defectors and blue dots denote cooperators.

\section{Results}

\subsection{The $P G G$ on a two-layer multiplex}

\subsubsection{The fixed cost per individual $P G G$}

The first set of results shows the mean frequency of cooperation across both layers plotted against the scaled enhancement factor $\eta=\frac{r}{\langle k\rangle+1}$ in the case of the fixed cost per individual (FCI) PGG for $m=2,\langle k\rangle=$ $3, \beta=0.5$ (figure 2 ).

Updating the strategies on each layer of the multiplex asynchronously leads to a higher frequency of players choosing the cooperative strategy within the network compared to updating synchronously (figure 2). At $\eta=0.76$ the difference is very large, with almost no cooperation present in the synchronous update system, and between 30 and $100 \%$ in the asynchronous update, with a similarly large difference seen for $\eta=0.78$. The value of $\eta$ at which the system changes from complete defection to mixed defection and cooperation is higher in the synchronous update case. Similarly, the value of $\eta$ at which the system changes from a mixture of strategies to complete cooperation is also higher in the case of the synchronous update. This demonstrates that asynchronous updating can act as a mechanism to maintain cooperation at lower enhancement factors that has not previously been considered.

To explain why there is a difference in the frequency of cooperation between the two update rules a cartoon of three players on the multiplex, $s, t$ and $u$, is shown in figures $3 \mathrm{a}$ and $3 \mathrm{~b}$. The strategies for player $s$ are $s_{i}$ on the top layer and $s_{j}$ on the bottom, and similarly for players $t$ and $u$.

On the top layer, player $s$ has selected player $u$ to imitate, and on the lower layer player $s$ will imitate player $t$. Assuming initially that $P_{u}>P_{s}$ and $P_{t}>P_{s}$, and that $\beta$ is not large, figure 3 a demonstrates the likely strategies after a single time step, with player $s$ imitating player $u$ on the top layer and player $t$ on the lower layer. When updating using the asynchronous rule the neighbours (and the payoffs) of the focal cooperator and defector may change before the cooperator has a chance to imitate the defector, resulting in a difference in imitation probability. If this probability changes by a large amount then player $s$ will no longer imitate player $t$ on the lower layer. Therefore updating the multiplex asynchronously rather than synchronously may lead to different frequencies of cooperation. A similar mechanism was found in reference [42], which found increased cooperation through strategy correlation across different network layers. 


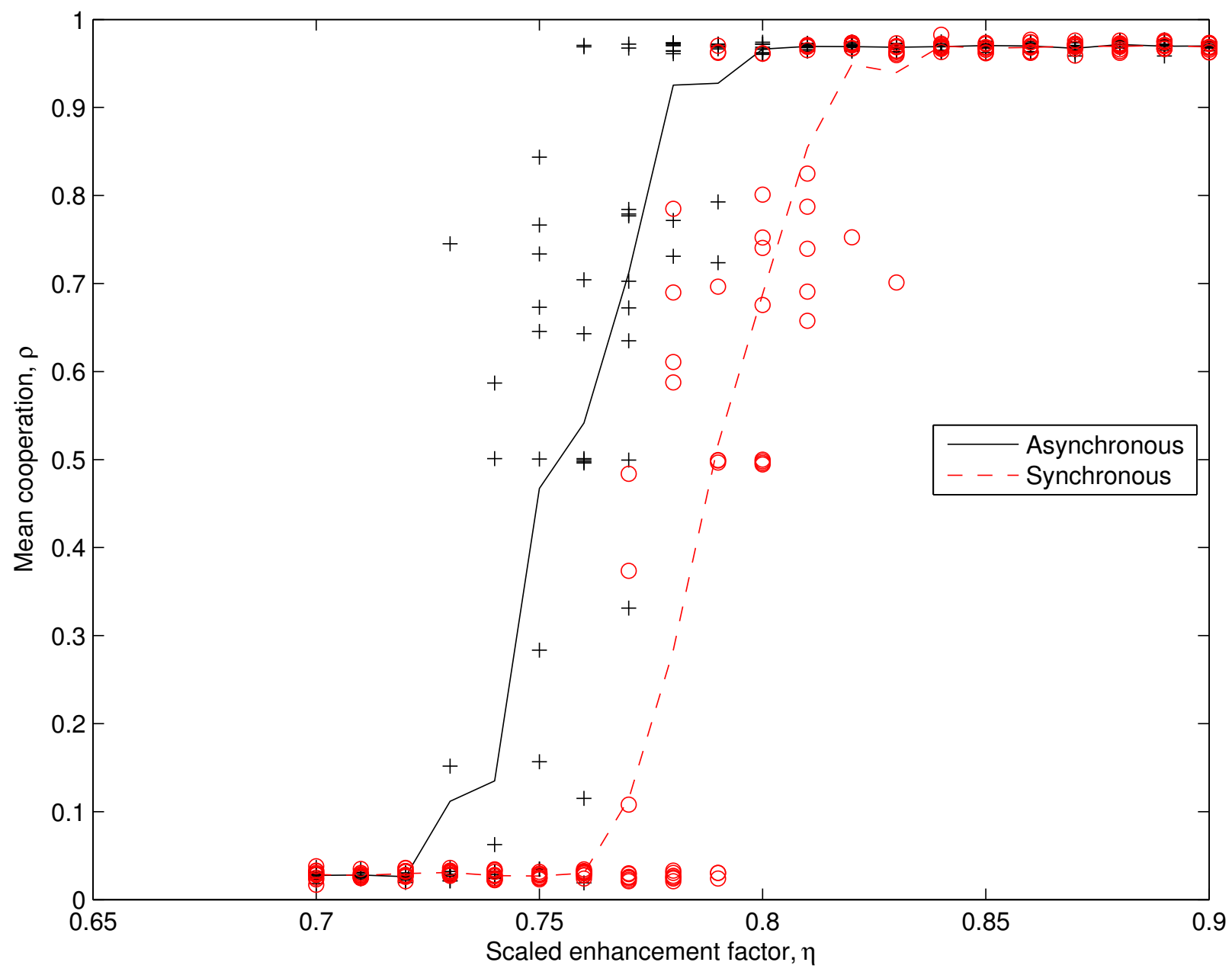

Figure 2: The frequency of cooperation on the two-layer PGG plotted against the scaled enhancement factor $\eta=\frac{r}{\langle k\rangle+1}$ for the asynchronous (solid black line) and synchronous (dashed red line) update rules for fixed cost per individual (FCI). $m=2, N=1000,\langle k\rangle=3, \beta=0.5$.

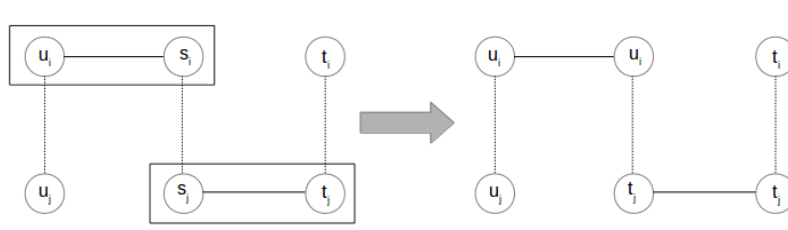

central node in the multiplex, $s$, imitates player $u$ in the (a) An example of the synchronous update rule. The cen- ${ }^{-}$first time step, but not player $t$ in the second update due
tral node in the multiplex, $s$, imitates player $u$ and $t$. to the change in payoffs. 


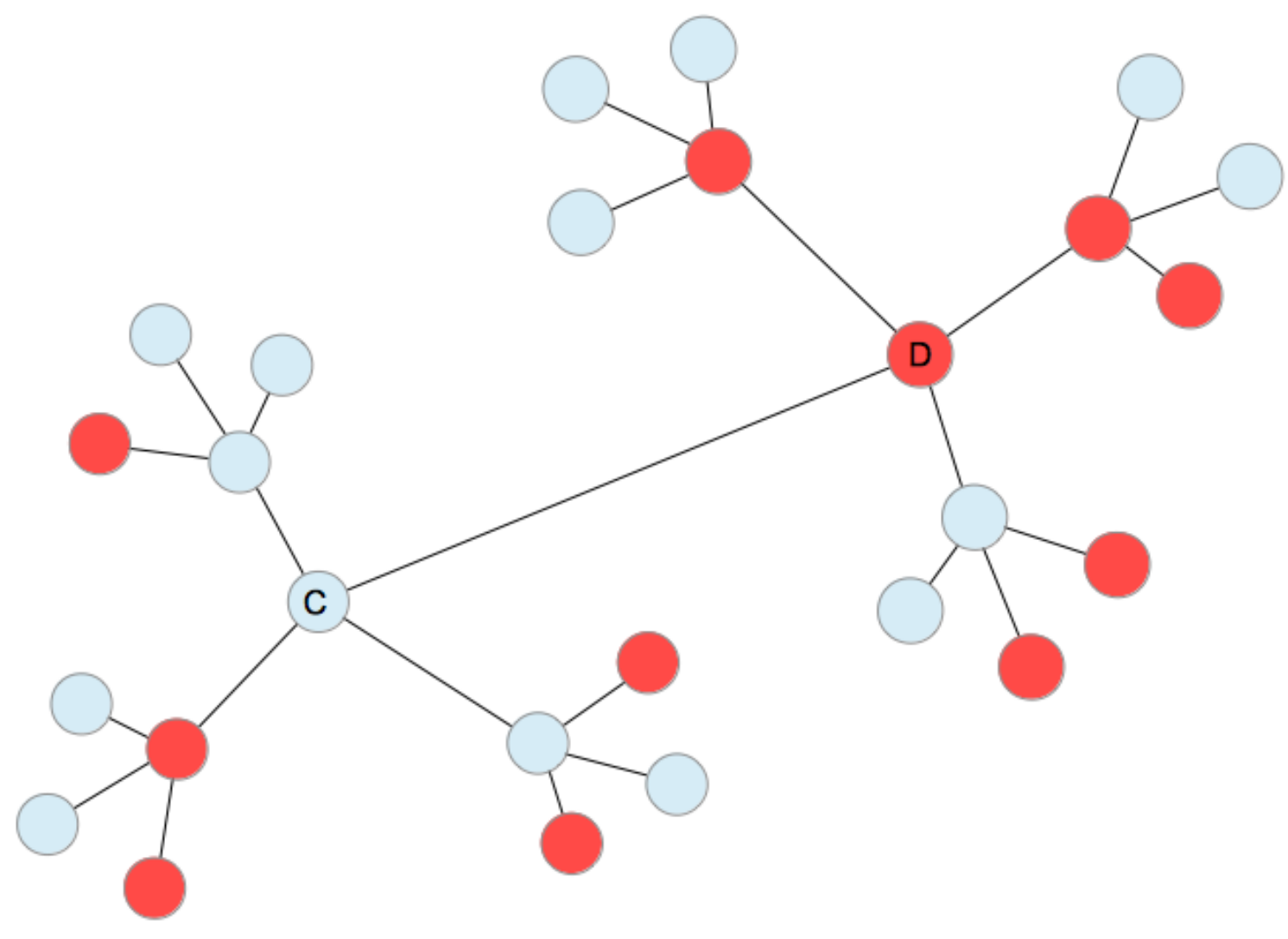

Figure 3: An example of a neighbouring cooperator and defector (labelled $\mathrm{C}$ and $\mathrm{D}$ respectively), and the composition of the neighbouring groups in which they accumulate payoffs (other cooperators coloured light blue, other defectors coloured dark red, colour online). 


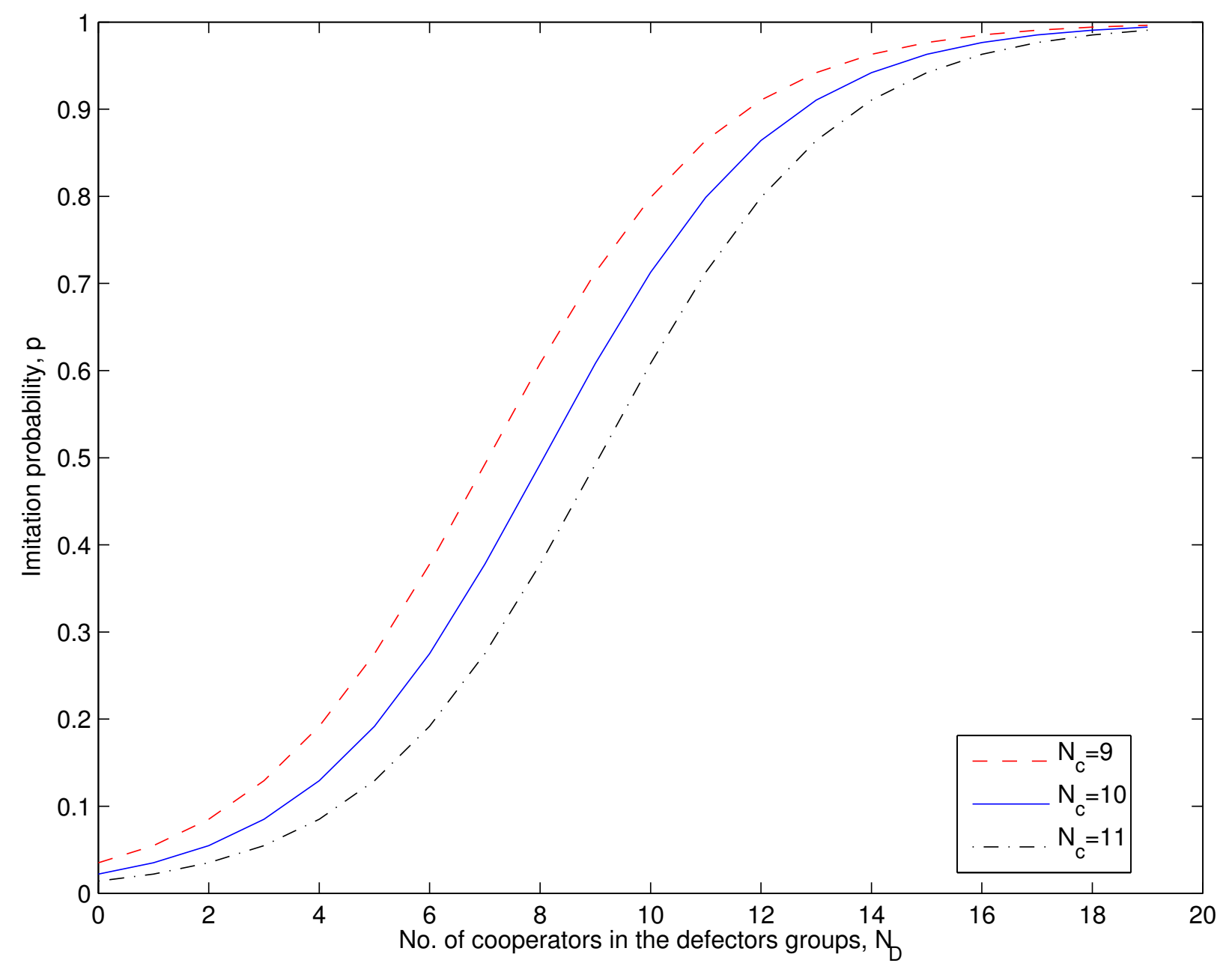

Figure 4: The probability of a cooperator in a neighbourhood composed of $N_{C}$ cooperators imitating a defector plotted against number of cooperators in the defector's neighbourhood, $N_{D}$ for the FCI PGG. $r=0.78, \beta=0.5,\langle k\rangle=3$.

We numerically calculate the expected change in probability by artificially forming neighbourhoods where a central cooperator and defector (labelled 'C' and ' $\mathrm{D}$ ' in figure 3) play in groups consisting of $N_{C}$ and $N_{D}$ cooperators respectively. The probability of a cooperator in a neighbourhood consisting of $N_{C}$ cooperators imitating a defector surrounded by $N_{D}$ cooperators is calculated using equation (3). Averaging these probabilities over all possible arrangements of $N_{C}$ and $N_{D}$ an expected imitation probability is calculated. Figure 4 plots this expected probability against $N_{D}$ for $N_{C}=9,10,11$ for the FCI PGG $(r=0.78, \beta=0.5,\langle k\rangle=3)$. This plot shows that if the group composition changes by a large amount, then the probability of a cooperator imitating a neighbouring defector will be significantly affected. Therefore, there is likely to be a large difference in the probability of a cooperator imitating a neighbouring defector under synchronous and asynchronous updating. 
These results are confirmed empirically by running a single iteration of the dynamics. Recording that player $i$ selects player $j$ to compare payoffs at time step $t$ enables the payoffs of the two players to be measured at both $t$ and $t-1$ (for $t>1$ ). Writing these payoffs as $P_{i}(t), P_{j}(t), P_{i}(t-1)$ and $P_{j}(t-1)$ and substituting them into equation (3) for both $t$ and $t-1$, the change in the probability of imitating a neighbouring strategy over two payoff updates can be measured. A histogram plotting the difference in imitation probabilities between two neighbours for consecutive time steps for the asynchronous rule is shown in figure 5 for $r=0.78, N=1000, \beta=0.5$.

In this histogram approximately $45 \%$ of the update probabilities change between each payoff update (in the synchronous case this number would be $0 \%$ ). Therefore, starting from identical networks with identical strategies, the probability of a given cooperator imitating a neighbouring defector (and vice versa) is altered when comparing the asynchronous and synchronous update rules. Because the largest change in group compositions occurs when the fraction of cooperation is approximately $50 \%$, this explains why the largest difference between the two rules is observed at this frequency of cooperation (figure 2).

To explain why cooperation is higher for the asynchronous update rule the probability of player $i$ imitating the strategy of neighbour $j$ for each payoff difference $P_{j}-P_{i}$ is empirically measured for both the asynchronous and synchronous update rules. We collected results from a single simulation for 1000 updates of the entire network, and calculated the probabilities by placing each measured payoff difference into a bin of width 0.1. Figure 6 shows the probability that a player imitates a neighbour's strategy plotted against the payoff difference, with the plot on the left showing the probability of a cooperator imitating a defector and the plot on the right showing the probability that a defector imitates a cooperator.

What is observed for both update rules (figure 6) is that for positive payoff differences the probability of strategy imitation is high, and increases as the difference increases, the reverse being true for negative payoff differences. In the case of the synchronous update rule (the dashed red line) the curve obeys equation (3) for most payoff differences, as expected. The probabilities become noisier at very low payoff differences, but this is due to the small number of empirical data points. In contrast the asynchronous rule (the solid black line) does not follow equation (3) particularly well for positive payoff differences. For most of the range of the positive payoff differences both the probability of a cooperator imitating a defector, and vice versa, is lower for each payoff difference compared to the results for the synchronous update rule (although at negative and very small positive payoff differences the two rules almost agree). In the asynchronous case a cooperator is less likely to imitate a defector than in the synchronous case, and this promotes the maintenance of cooperation throughout the network. Hence, the asynchronous and synchronous update rules lead to different frequencies of cooperation in the population due to the additional payoff update in each time step in the asynchronous case, and this leads to a reduced probability of a player imitating a better performing neighbour. In the parameter range investigated here $(\eta<1)$ in the PGG a better performing neighbour is likely to be a defector, and so the frequency of cooperation is likely to be higher in 

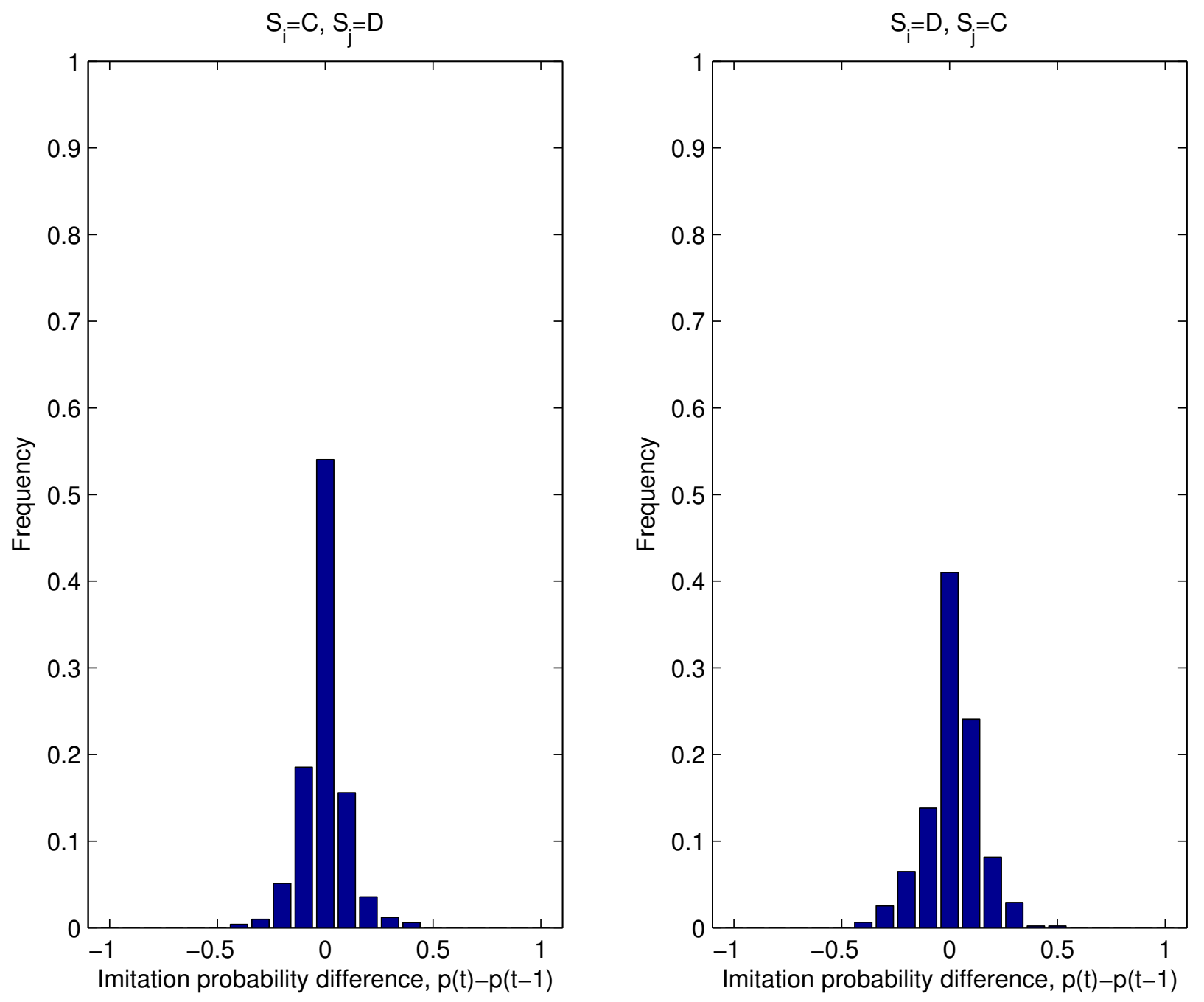

Figure 5: Histogram showing the change in imitation probabilities between players $i$ and $j$ after a single update of the payoffs for $s_{i}=C, s_{j}=D$ (left) and $s_{i}=D, s_{j}=C$ (right) for the FCI PGG. $r=0.78, m=$ $2, N=1000, \beta=0.5$ 

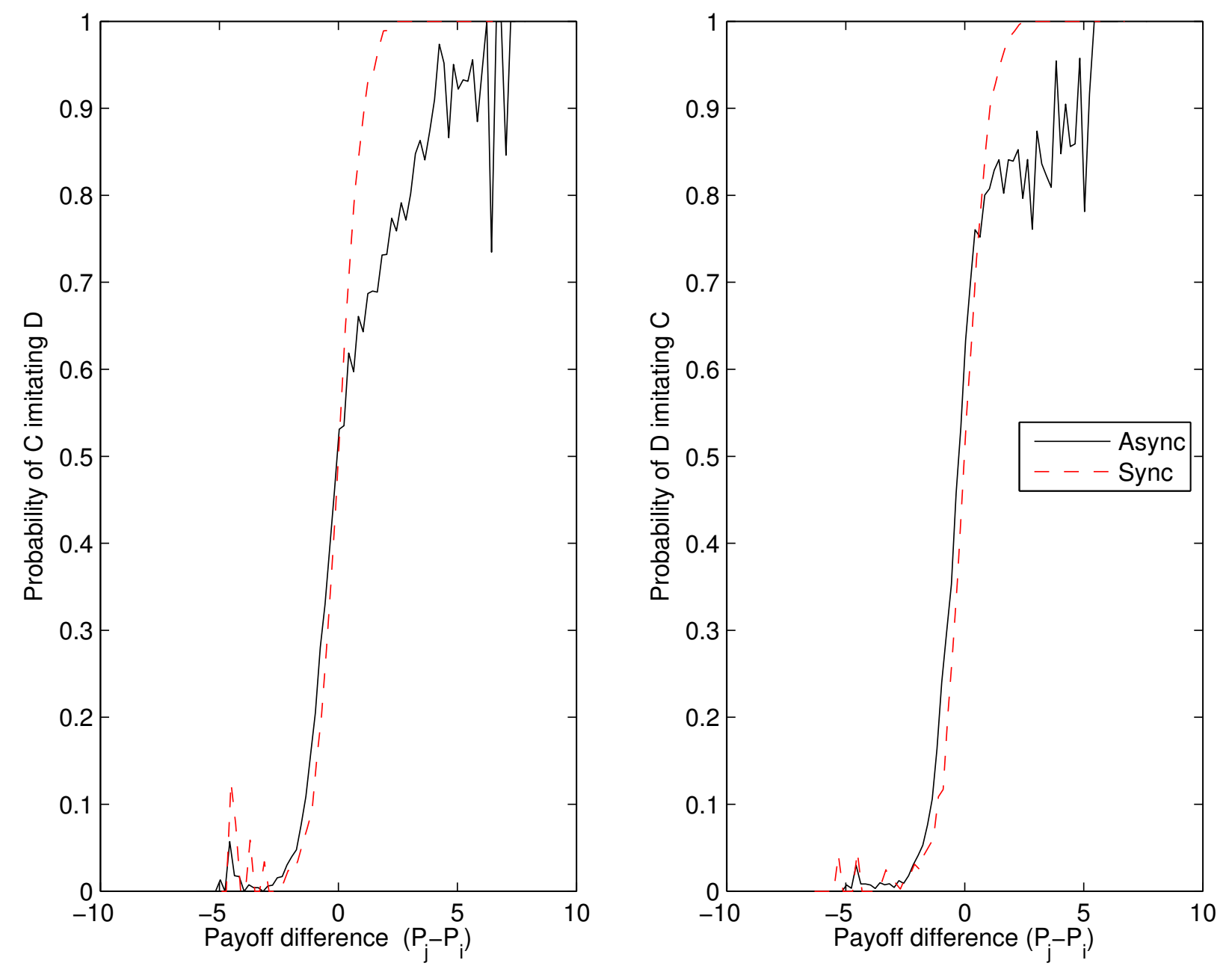

Figure 6: The probability of strategy imitation on the two-layer multiplex for the asynchronous (solid black line) and synchronous (dashed red line) update rules for fixed cost per individual (FCI). $N=1000, m=$ $2, \eta=0.76,\langle k\rangle=3, \beta=0.5$. 


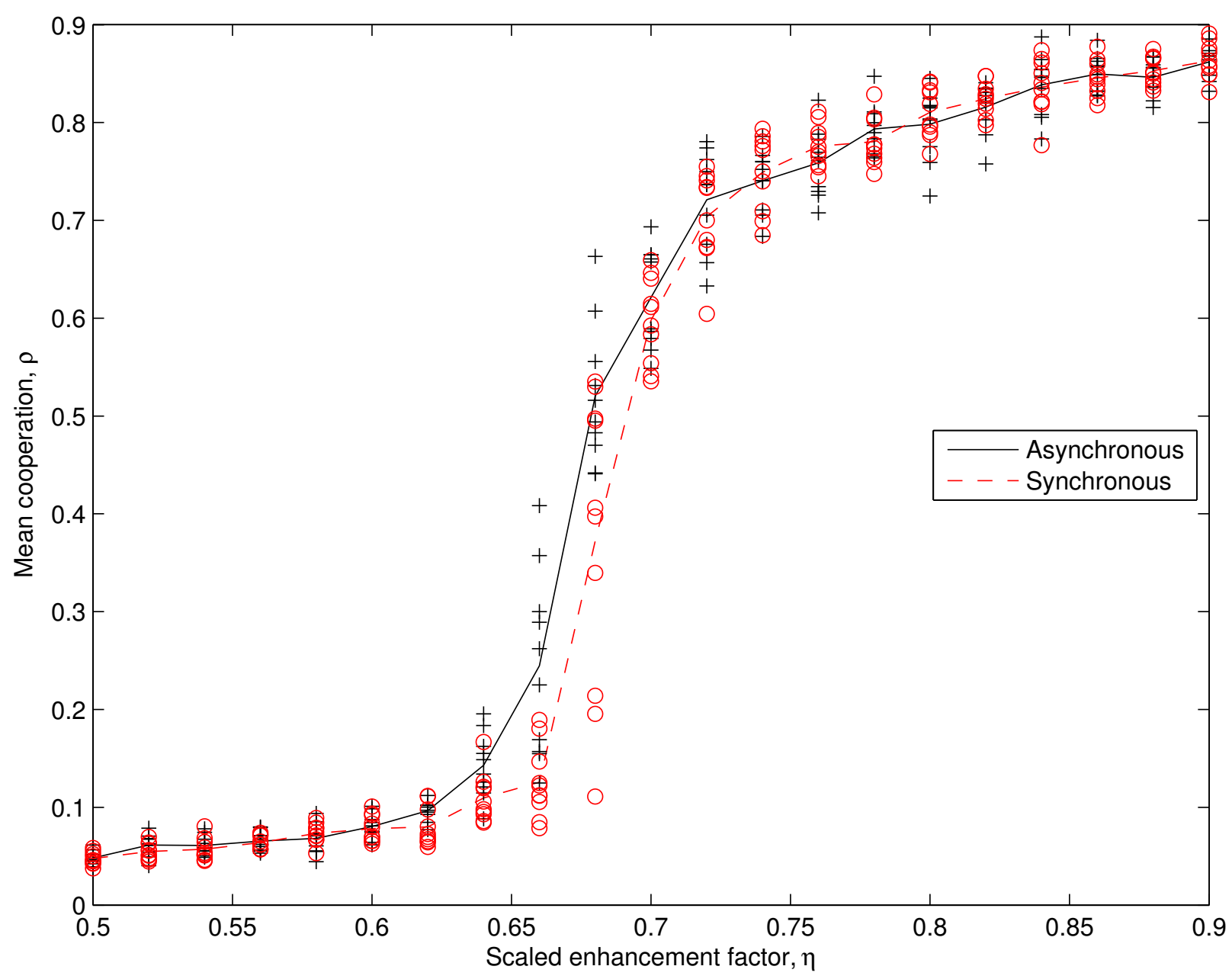

Figure 7: The frequency of cooperation on the two-layer PGG plotted against the scaled enhancement factor $\eta=\frac{r}{\langle k\rangle+1}$ for the asynchronous (solid black line) and synchronous (dashed red line) update rules for fixed cost per individual (FCI). $N=1000, m=2,\langle k\rangle=3, \beta=0$.

the asynchronous case. This also explains the asymmetry between the asynchronous results in each plot, as cooperators are less likely to imitate better performing defectors.

\subsection{Additional results on the $P G G$}

3.2.1. Deterministic $(\beta=0)$ dynamics for the fixed cost per individual $P G G$

Testing the importance of the rule for other values of $\beta$, figure 7 shows that for FCI deterministic updates $(\beta=0)$ the asynchronous update rule once again leads to a higher final frequency of cooperation for a range of enhancement factors. However, the differences in figure 7 are much smaller than in figure 2, with some asynchronous and synchronous results overlapping for the entire range of $\eta$. 


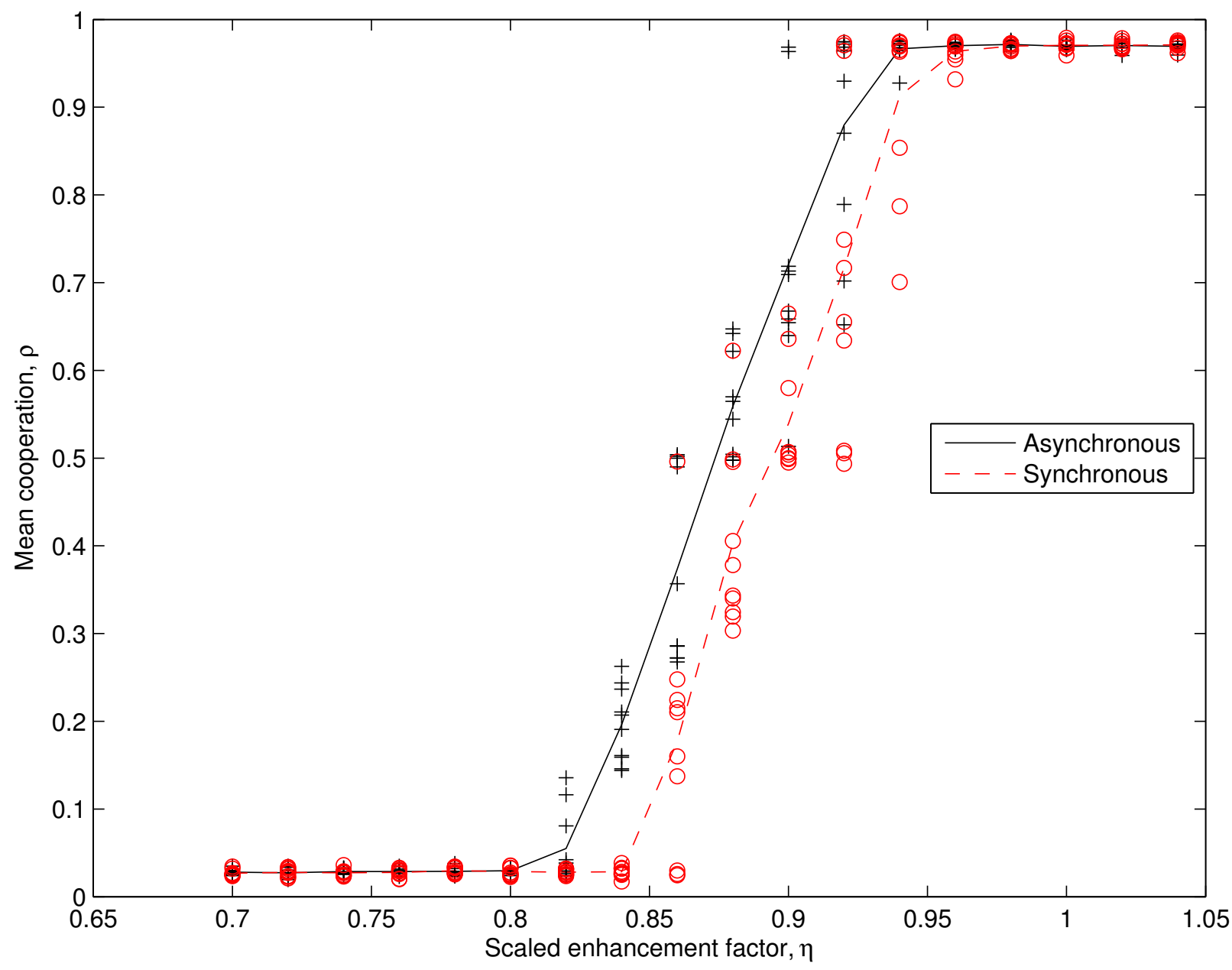

Figure 8: The frequency of cooperation on the two-layer PGG plotted against the scaled enhancement factor $\eta=\frac{r}{\langle k\rangle+1}$ for the asynchronous (solid black line) and synchronous (dashed red line) update rules for fixed cost per group (FCG). $N=1000, m=2,\langle k\rangle=3, \beta=0.5$. 


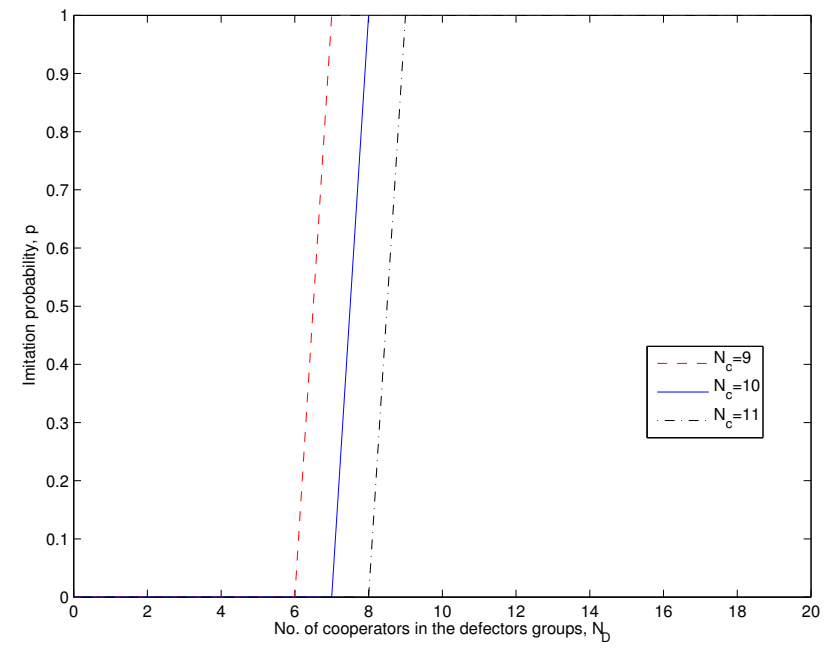

(a) FCI $r=0.72, \beta=0$

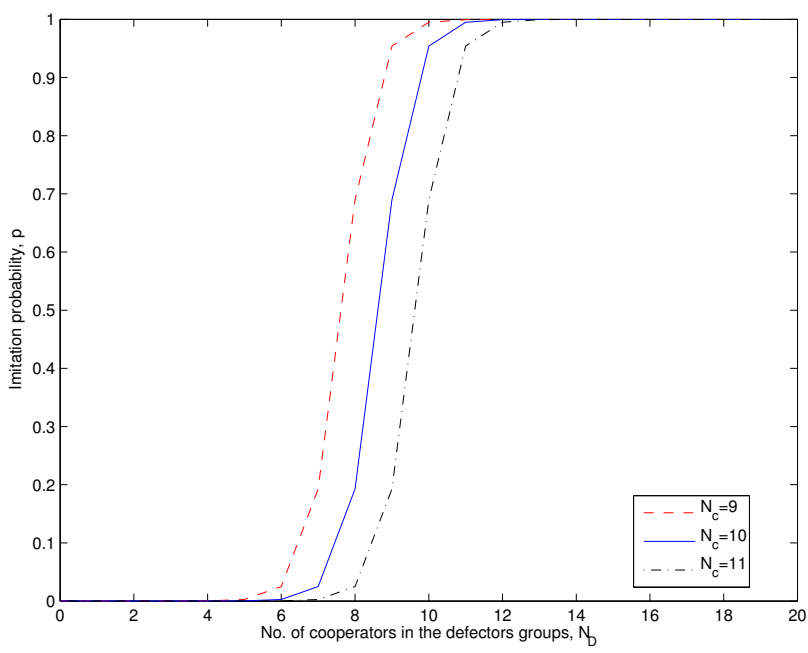

(b) FCG $r=0.9, \beta=0.5$

Figure 9: The probability of a cooperator in a neighbourhood composed of $N_{C}$ cooperators imitating a defector plotted against number of cooperators in the defector's neighbourhood, $N_{D}$ for the FCI PGG $(r=0.72, \beta=0)$ and the FCG PGG $(r=0.9, \beta=0.5) .\langle k\rangle=3$.

\subsubsection{The fixed cost per group $P G G$}

Results for the fixed cost per group (FCG) PGG are presented in this section. Figure 8 shows the effect of the choice of update rule when the players contribute the same to each group (FCG) in the PGG. Once again, in general the asynchronous update rule leads to higher frequencies of cooperation for a wide range of $\eta$ (figure 8). However, comparing these results to the FCI case in figure 2 the choice of update rule makes less of a difference to both the final frequency of cooperation and the enhancement factor at which the system produces a mixed state of cooperators and defectors or fixates to a single strategy.

\subsection{Comparison of imitation probabilities for FCI $\beta=0$ and FCG $\beta=0.5$}

We repeated the calculations for imitation probabilities performed in section 3.1 for the FCI with no noise $(\beta=0)$ and present the results in figure 9a. The imitation probabilities are also calculated for the FCG PGG $(\beta=0.5)$ and the results shown in figure 9b. Comparing these results with those for the FCI $(\beta=0.5)$, the imitation probability curves in figures $9 \mathrm{a}$ and $9 \mathrm{~b}$ are much steeper compared to those in figure 4. Therefore, if the number of cooperators in a group changes between payoff updates this has less of an effect on the probability of strategy imitation, and so the choice of update rule will have less of an effect on the final frequency of cooperation.

These results are confirmed by the empirical measurements of the change in imitation probability in figure 10 for the FCI PGG $(\beta=0)$ and the FCG PGG $(\beta=0.5)$. Comparing the frequency of $p(t)-p(t-1) \neq 0$ for the FCI $(\beta=0)$ (figure 10a) and the FCG $(\beta=0.5)$ (figure 10b) with the results for the noisy FCI $(\beta=0.5)$ (figure 5$)$, the scenario with the largest imitation probability change is the FCI PGG $(\beta=0.5)$, 

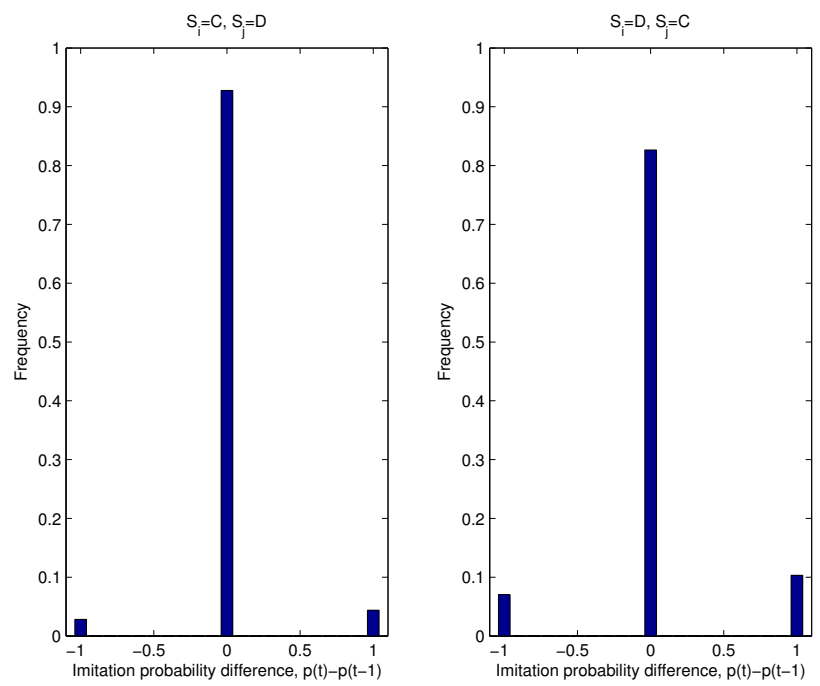

(a) FCI $r=0.72, N=1000, \beta=0$
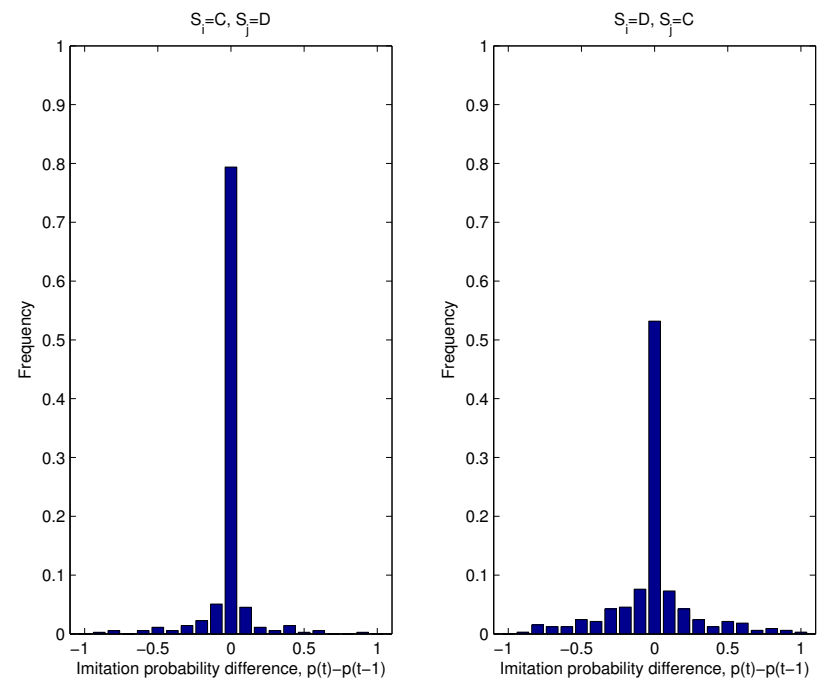

(b) FCG $r=0.9, N=1000, \beta=0.5$

Figure 10: Histogram showing the change in imitation probabilities between players $i$ and $j$ after a single update of the payoffs for $s_{i}=C, s_{j}=D$ and $s_{i}=D, s_{j}=C$ for the PGG. Figure 10a FCI $r=0.72, N=$ $1000, \beta=0$, figure 10b FCG $r=0.9, m=2, N=1000, \beta=0.5$. 
followed by FCG $(\beta=0.5)$ and finally FCI $(\beta=0)$. Therefore, the choice of update rule will have the most impact for the FCI $(\beta=0.5)$, as here the probability of imitating a neighbouring defector changes the most between payoff updates. What these results show is that when imitation probabilities are strongly dependent on the composition of the groups in which the players outcomes will be significantly affected by the choice of update rule.

\subsection{The PD on a two-layer multiplex}

In this section we consider another common game used to model cooperation, namely the prisoner's dilemma (PD). In the PD the game is played in pairs rather than groups, and the payoff for each player is the sum of the payoffs in each of these pairwise interactions. As in the PGG the neighbours in the network denote who each player plays against, and the total payoff for each player is found by summing over each of the pairwise interactions across all of the layers. The standard way to represent the payoffs gained is in the form of a matrix, where the rows define the strategy chosen by player one, and the columns the strategy chosen by player two, with the values in the matrix giving the payoffs allocated to each player.

Table 1: The payoffs for the pairwise game.

\begin{tabular}{c|c|c}
\hline & $\mathrm{C}$ & $\mathrm{D}$ \\
\hline $\mathrm{C}$ & $\mathrm{R}, \mathrm{R}$ & $\mathrm{S}, \mathrm{T}$ \\
$\mathrm{D}$ & $\mathrm{T}, \mathrm{S}$ & $\mathrm{P}, \mathrm{P}$
\end{tabular}

In the PD the payoffs are ordered $T>R>P>S$, and often this is simplified to $T=b, R=1, P=S=0$, where $b>1$ is the parameter of interest and is labelled as the 'temptation to defect'.

Figure 11 shows results for the PD run on a two-layer Erdős-Rényi multiplex network, for parameters $N=1000,\langle k\rangle=3$. The dynamics in these simulations are very similar to those in section 3.1 , with each player selecting a neighbour at random and comparing payoffs. When examining the PD on networks two standard update rules are investigated. In figure 11a the Fermi imitation rule (equation (3)) is used in order to decide if player $i$ should imitate player $j$. In the original model of cooperation on a multiplex (see reference [35]) strategy imitation occurs with probability

$$
p\left(s_{i} \rightarrow s_{j}\right)=\frac{P_{j}-P_{i}}{K_{\max } b}
$$

where $P_{i}$ is the payoff of player $i$ as usual, $K_{\max }$ is the largest sum of the degrees of player $i$ and $j$ across all of the layers, and $b$ is the temptation to defect. We show that the choice of update rule also alters the frequency of cooperation if this different imitation rule is used (figure 11b)

Figure 11 shows the final frequencies of cooperation for the asynchronous and synchronous update rules for these two different imitation rules. For Fermi imitations (Figure 11a) results are very similar to those found in section 3.1: for $1.61<b<1.86$ the asynchronous update rule leads to a higher frequency of 


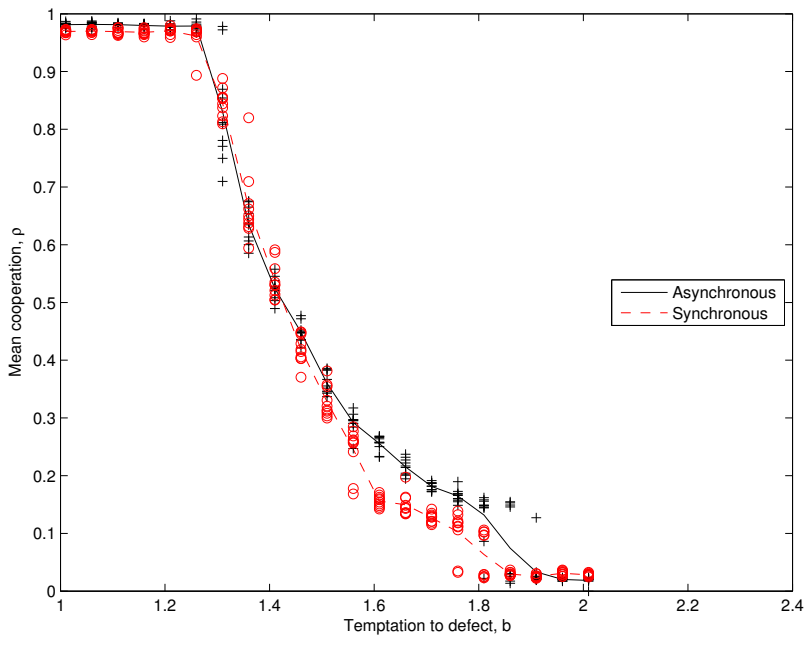

(a) The Fermi imitation rule. $\beta=0.1$.

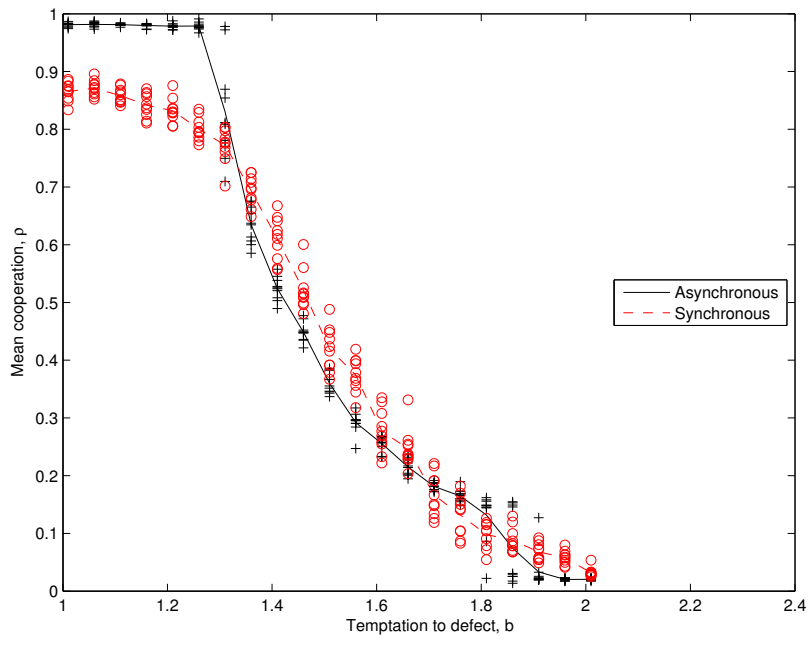

(b) The payoff difference update rule

Figure 11: The frequency of cooperation on the two-layer PD plotted against the temptation to defect for the asynchronous (solid black line) and synchronous (dashed red line) update rules for the Fermi imitation rule $(\beta=0.1) 11 \mathrm{a}$ and the payoff difference update rule 11b. $N=1000, m=2,\langle k\rangle=3$.

cooperation compared to the synchronous rule, although the effect is not large. When imitation probabilities follow equation (4) there is a significant difference between the two rules for low $b$, and then little difference as the temptation to defect increases.

This difference is explained by plotting the imitation probabilities for $b=1.1$ for both the Fermi and the payoff difference update rules. For $b=1.1$ the frequency of cooperation is high, and so the likely number of cooperators in the neighbouring defector's group is large. Figure 12a shows that in the Fermi imitation rule there is no difference between the different number of cooperators. Therefore, if the number of cooperators in the group changes through the additional strategy update in the asynchronous case, this will not lead to a large difference in imitation probability. This is not what is observed in figure $12 \mathrm{~b}$, where there is a difference in the imitation probability between each value of $N_{D}$. Therefore, if the number of cooperators in a group changes, this will lead to a different imitation probability.

\section{Discussion and conclusion}

We have shown that the asynchronous update rule consistently leads to higher frequencies of cooperation over a range of enhancement factors in the public goods game, and can also increase cooperation in the prisoner's dilemma. Through numerical simulations we find that the strength of the effect depends on a number of factors, including the type of game played, the noise and the strategy imitation rule. In each of these cases when the probability of a player of one strategy imitating another is highly sensitive to changes in the player's neighbourhood, the choice of asynchronous or synchronous update rule has more of an effect on the final frequency of cooperation. The public goods game is most sensitive to changes in the neighbourhood 


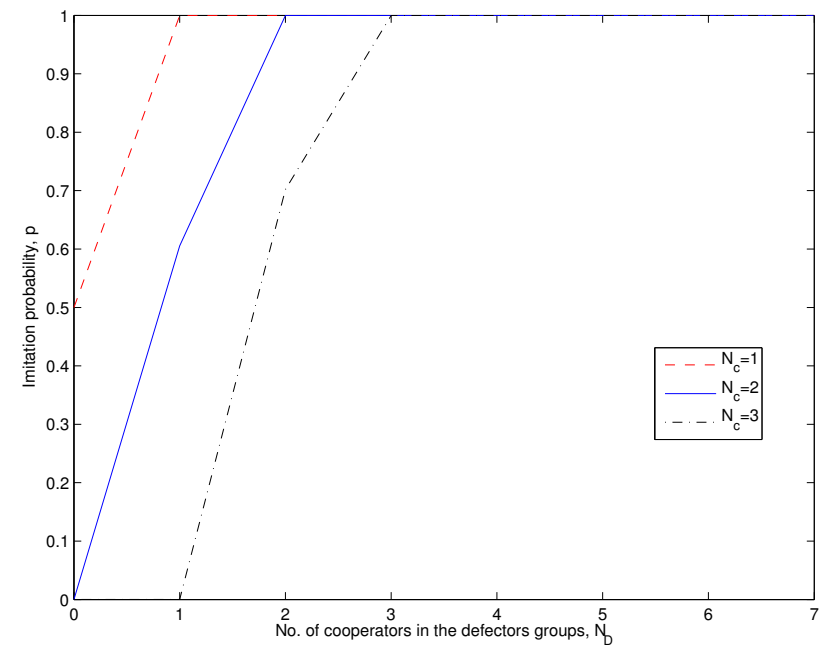

(a) Fermi imitation rule.

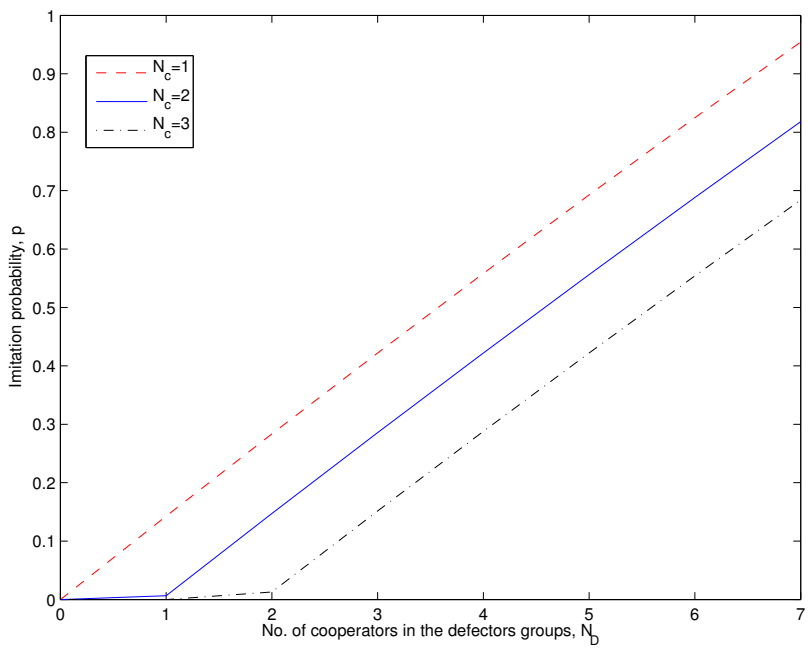

(b) PD payoff difference imitation rule.

Figure 12: The probability of a cooperator in a neighbourhood composed of $N_{C}$ cooperators imitating a defector plotted against number of cooperators in the defector's neighbourhood, $N_{D}$ for the PD Fermi imitation and the PD payoff difference update. $b=1.1,\langle k\rangle=3$.

when a player divides a single unit between its neighbours (the FCI PGG), compared to when a single unit is donated to all (the FCG PGG). Therefore, the choice of rule is more significant for the FCI PGG. In contrast, the prisoner's dilemma is not as sensitive to changes in the neighbourhood, and so the effect is much less pronounced. A single change in a player's neighbourhood will alter the probability of strategy imitation by large or small amounts depending on the strategy imitation rule chosen. If the payoff difference imitation rule is used the probability is highly dependent on the neighbourhood, whereas the Fermi imitation rule is much less sensitive. If the Fermi rule has a large noise parameter, this makes it more sensitive to a change in the neighbourhood.

We conclude that if the strategy imitation rule is strongly payoff dependent, and if the average payoffs of the game on the multiplex are greatly affected by the number of cooperators in a group, then updating layers asynchronously will lead to an increase in cooperation for a wide parameter range. This suggests that in order to model the evolution of cooperation robustly on multiplex networks, we must choose an updating strategy that most accurately captures the dynamics of the real-world scenario we hope to model.

In general, updating layers asynchronously rather than synchronously increases the amount of cooperation on a multiplex network. This is because the probability of one strategy imitating another is sensitive to the composition of a player's group. The more sensitive the probability of one player imitating another is to the composition of the neighbourhood, the larger the difference in cooperation between the two rules. The choice of asynchronous or synchronous updates also suggests a method of increasing cooperation on multilayer systems, namely to slow down the rate of updating the strategies on each layer compared to the 
rate at which the payoffs are calculated. Specifically, this can be achieved with a move to asynchronous updates rather than synchronous.

\section{Acknowledgements}

This work was supported by the Engineering and Physical Sciences Research Council [grant number EP/H021779/1] (Evolution and Resilience of Industrial Ecosystems, ERIE).

[1] J. Ledyard. Public Goods: A Survey of Experimental Research. In J Roth and A Kagel, editors, The Handbook of Experimental Economics. Princeton University Press, Princeton, 1995.

[2] Jelena Grujić, Carlos Gracia-Lázaro, Manfred Milinski, Dirk Semmann, Arne Traulsen, José A Cuesta, Yamir Moreno, and Angel Sánchez. A comparative analysis of spatial Prisoner's Dilemma experiments: Conditional cooperation and payoff irrelevance. Scientific reports, 4:4615, 2014.

[3] Juan Camilo Cardenas. Social Norms and Behavior in the Local Commons as Seen Through the Lens of Field Experiments. Environmental and Resource Economics, 48(3):451-485, 2011.

[4] Ernst Fehr and Andreas Leibbrandt. A field study on cooperativeness and impatience in the Tragedy of the Commons. Journal of Public Economics, 95(9-10):1144-1155, 2011.

[5] Martin A Nowak. Five rules for the evolution of cooperation. Science (New York, N.Y.), 314(5805):1560-3, Dec 2006.

[6] R Axelrod. The Evolution of Cooperation. Basic Books, Inc., New York, 1984.

[7] M Nowak and R May. Evolutionary games and spatial chaos. Nature, 359:826-829, 1992.

[8] F. Santos and J. Pacheco. Scale-Free Networks Provide a Unifying Framework for the Emergence of Cooperation. Physical Review Letters, 95(9):098104, Aug 2005.

[9] F Santos, M Santos, and J Pacheco. Social diversity promotes the emergence of cooperation in public goods games. Nature, 454(July), 2008.

[10] György Szabó, Jeromos Vukov, and Attila Szolnoki. Phase diagrams for an evolutionary prisoner's dilemma game on two-dimensional lattices. Physical Review E, 72(4):047107, Oct 2005.

[11] Attila Szolnoki, Matjaž Perc, and György Szabó. Topology-independent impact of noise on cooperation in spatial public goods games. Physical Review E, 80(5):056109, Nov 2009.

[12] Christoph Hauert and Michael Doebeli. Spatial structure often inhibits the evolution of cooperation in the snowdrift game. Nature, 428:2-5, 2004.

[13] M Ali Saif and Prashant M Gade. The prisoner's dilemma with semi-synchronous updates: evidence for a first-order phase transition. Journal of Statistical Mechanics: Theory and Experiment, 2009:P07023, 2009.

[14] Hannelore Brandt, Christoph Hauert, and Karl Sigmund. Punishment and reputation in spatial public goods games. Proceedings. Biological sciences / The Royal Society, 270(1519):1099-104, May 2003.

[15] Dirk Helbing, Attila Szolnoki, Matjaž Perc, and György Szabó. Punish, but not too hard: how costly punishment spreads in the spatial public goods game. New Journal of Physics, 12(8):083005, Aug 2010.

[16] György Szabó and Christoph Hauert. Phase Transitions and Volunteering in Spatial Public Goods Games. Physical Review Letters, 89(11):118101, Aug 2002.

[17] Matjaž Perc and Attila Szolnoki. Social diversity and promotion of cooperation in the spatial prisoner's dilemma game. Physical Review E, 77(1):011904, Jan 2008.

[18] Sergey V Buldyrev, Roni Parshani, Gerald Paul, H Eugene Stanley, and Shlomo Havlin. Catastrophic cascade of failures in interdependent networks. Nature, 464(7291):1025-8, Apr 2010.

[19] Charles D Brummitt, Raissa M D'Souza, and E a Leicht. Suppressing cascades of load in interdependent networks. Proceedings of the National Academy of Sciences of the United States of America, 109(12):E680-9, Mar 2012.

[20] Eugenio Valdano, Luca Ferreri, Chiara Poletto, and Vittoria Colizza. Analytical computation of the epidemic threshold on temporal networks. Physical Review X, 5(2):19, 2015.

[21] Alessio Cardillo, Jesús Gómez-Gardeñes, Massimiliano Zanin, Miguel Romance, David Papo, Francisco del Pozo, and Stefano Boccaletti. Emergence of network features from multiplexity. Scientific reports, 3:1344, 2013.

[22] S. Gómez, a. Díaz-Guilera, J. Gómez-Gardeñes, C. J. Pérez-Vicente, Y. Moreno, and a. Arenas. Diffusion Dynamics on Multiplex Networks. Physical Review Letters, 110(2):028701, Jan 2013.

[23] Mikko Kivelä, Alex Arenas, Marc Barthelemy, James P. Gleeson, Yamir Moreno, and Mason A. Porter. Multilayer networks. Journal of Complex Networks, 2(3):203-271, 2014.

[24] Jesús Gómez-Gardeñes, Carlos Gracia-Lázaro, Luis Mario Floría, and Yamir Moreno. Evolutionary dynamics on interdependent populations. Physical Review E, 86(5):56113, Nov 2012.

[25] Luo-Luo Jiang and Matjaž Perc. Spreading of cooperative behaviour across interdependent groups. Scientific Reports, 3(2483), 2013.

[26] Zhen Wang, Attila Szolnoki, and Matjaž Perc. Evolution of public cooperation on interdependent networks: The impact of biased utility functions. EPL (Europhysics Letters), 97(4):48001, Feb 2012.

[27] Zhen Wang, Attila Szolnoki, and Matjaž Perc. Interdependent network reciprocity in evolutionary games. Scientific reports, 3:1183, Jan 2013. 
[28] Xiaokun Meng, Shiwen Sun, Xiaoxuan Li, Li Wang, Chengyi Xia, and Junqing Sun. Interdependency enriches the spatial reciprocity in prisoner's dilemma game on weighted networks. Physica A: Statistical Mechanics and its Applications, 442:388-396, 2016.

[29] Zhen Wang, Attila Szolnoki, and Matjaž Perc. Optimal interdependence between networks for the evolution of cooperation. Scientific reports, 3:2470, Jan 2013.

[30] Baokui Wang, Xiaojie Chen, and Long Wang. Probabilistic interconnection between interdependent networks promotes cooperation in the public goods game. Journal of Statistical Mechanics: Theory and Experiment, 2012(11):P11017, Nov 2012.

[31] Luo-Luo Jiang, Wen-Jing Li, and Zhen Wang. Multiple effect of social influence on cooperation in interdependent network games. Scientific Reports, 5:14657, 2015.

[32] Chao Luo, Xiaolin Zhang, Hong Liu, and Rui Shao. Cooperation in memory-based prisoner's dilemma game on interdependent networks. Physica A: Statistical Mechanics and its Applications, 450:560-569, 2016.

[33] Baokui Wang, Zhenhua Pei, and Long Wang. Evolutionary dynamics of cooperation on interdependent networks with the Prisoner's Dilemma and Snowdrift Game. EPL (Europhysics Letters), 107(5):58006, 2014.

[34] Zhen Wang, Lin Wang, and Attila Szolnoki. Evolutionary games on multilayer networks: A colloquium. European Physical Journal B, 88(124):1-14, 2015.

[35] Jesús Gómez-Gardeñes, Irene Reinares, Alex Arenas, and Luis Mario Floría. Evolution of cooperation in multiplex networks. Scientific reports, 2:620, Jan 2012.

[36] Joan T Matamalas, Julia Poncela-Casasnovas, Sergio Gómez, and Alex Arenas. Strategical incoherence regulates cooperation in social dilemmas on multiplex networks. Scientific Reports, 5:9519, 2015.

[37] Z Wang, Lin Wang, and M Perc. Degree mixing in multilayer networks impedes the evolution of cooperation. Physical Review E, 052813:1-8, 2014

[38] M D Santos, S N Dorogovtsev, and J F F Mendes. Biased imitation in coupled evolutionary games in interdependent networks. Scientific reports, 4:1-19, 2014.

[39] Alessandro Di Stefano, Marialisa Scatà, Aurelio La Corte, Pietro Liò, Emanuele Catania, Ermanno Guardo, and Salvatore Pagano. Quantifying the Role of Homophily in Human Cooperation Using Multiplex Evolutionary Game Theory. Plos One, 10(10):e0140646, 2015.

[40] Yanling Zhang, Feng Fu, Xiaojie Chen, Guangming Xie, and Long Wang. Cooperation in group-structured populations with two layers of interactions. Scientific Reports, 5:17446, 2015.

[41] A Mukherji, V Rajan, and J R Slagle. Robustness of cooperation. Nature, 379:125-126, 1996.

[42] Attila Szolnoki and Matjaž Perc. Information sharing promotes prosocial behaviour. New Journal of Physics, $15,2013$. 